\title{
Investigation on the PAPR performance of odd-bit QAM constellations for DFT spread OFDM systems
}

\author{
Ahmed M. Sana, Amer T. Saeed, Yaseen Kh. Yaseen \\ College of Petroleum Processes Engineering, Tikrit University, Iraq
}

\section{Article Info}

Article history:

Received Apr 20, 2020

Revised Jun 23, 2020

Accepted Jul 8, 2020

\section{Keywords:}

Cross QAM

DFT-S-OFDM

Odd bit QAM

PAPR

Rectangular QAM

\begin{abstract}
Adaptive quadrature amplitude modulation (QAM) is a crucial scheme that enables the modern communication systems to overcome the adverse effects of channel fluctuations and maintain an acceptable spectral efficiency. In order to enhance adaptive modulation even further, adoption of odd-bit QAM constellations alongside even constellations had been suggested to improve the transmission efficiency of adaptive QAM modulation. Hence, odd-bit QAM had been extensively studied, analyzed, and tested by many researchers for various patterns, sizes, and communication systems in terms of bit error rate (BER) and peak to average power ratio (PAPR). However, the PAPR performance of odd-bit QAM constellation with single carrier transmission systems adopted in the uplink of the $4 \mathrm{G}$ long term evolution (LTE) standards caught almost no research interest. In this paper, the PAPR performance of both cross and rectangular odd-bit QAM constellations are investigated for DFT-S-OFDM systems. Complementary cumulative distribution functions (CCDFs) and probability density functions (PDFs) curves for PAPR are also obtained. Finally, an equation for PAPR PDF is empirically derived for odd-bit cross QAM based DFT-S-OFDM. The results show that cross odd-bit QAM outperforms the rectangular odd-bit QAM in terms of PAPR by $1.02 \mathrm{~dB}$ for 8-QAM and $1.3 \mathrm{~dB}$ for 32-QAM. This proves that cross odd-bit QAM is a better choice in terms of PAPR for DFT-SOFDM systems.
\end{abstract}

This is an open access article under the CC BY-SA license.

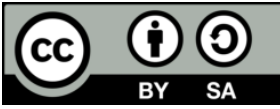

\section{Corresponding Author:}

Ahmed M. Sana

College of Petroleum Processes Engineering

Tikrit University

Samarra, Saladdin Province, Iraq

Email: ahmed.mohammed@tu.edu.iq

\section{INTRODUCTION}

Orthogonal frequency division multiplexing (OFDM) system is a popular multi-access technique for its spectral efficiency, and tolerance to multipath fading and inter-symbol interference. However, its main drawback is the high PAPR that manifests itself as high peaks in OFDM signal driving the power amplifiers out of linearity and causing an intermodulation distortion, especially in the uplink. One of the famous solutions to reduce the high PAPR problem in OFDM systems is precoding the data symbols using a discrete fourier transform (DFT) block before feeding them to the conventional OFDM modulator. DFT precoding process will largely reduce the higher signal peaks resulting in a PAPR nearly the same as the input data symbols. Hence, DFT-S-OFDM system (also known as single carrier frequency division multiple access (SCFDMA) system) is used as a multi-access technique for the uplink LTE and LTE-Advanced 4G standards.

On the other hand, in order to enable the communication systems to maintain an acceptable performance in varying channel conditions, it is important to employ adaptive QAM modulation scheme to 
control the data transmission rate via controlling the constellation size according to the channel conditions. Currently, the even $2^{2 \mathrm{n}}$ ( $\mathrm{n}$ is an integer) QAM constellations is predominant in the most communication systems. Although the BER performance of odd $2^{2(n+1)}$ QAM constellation outperforms even QAM constellations providing better efficiency for adaptive modulation [1], it is less favorable due to the unbalanced average power between the real and imaginary signals and due to the high PAPR of rectangular odd-bit QAM constellations. Cross odd-bit QAM, though, cannot adopt the gray labelling which making its bit error rate (BER) performance suboptimal compared to the rectangular constellation, it has a balanced average power between real and imaginary signals and better PAPR performance making it a favorable candidate to adopt in adaptive modulation.

PAPR problem in communications systems had been an interesting topic for many researchers. Numerous articles focused on the analysis and reduction techniques for PAPR problem in various communication systems. An exact PAPR distribution was derived for band-limited OFDM systems and an approximate closed-form expression for PAPR distribution was proposed in [2]. PAPR distribution for OFDM systems was calculated and derived based on the extreme value theory in [3] and [4]. The performance analysis of many PAPR reduction techniques for OFDM and SC-FDMA systems were presented in [5-11].

On the other hand, Analysis of the PAPR performance in OFDM and SC-FDMA systems also caught a big interest for many researchers. Authors in [12-13] analyzed the effect of subcarrier mapping and pulse shaping on the PAPR performance of SC-FDMA systems. In [14-15], PAPR was analyzed for OFDM and beamforming MIMO-OFDM systems and mitigation algorithms were proposed to solve the deterioration of PAPR performance caused by OFDM and beamforming. Artificial intelligence was exploited in [16] for analyzing the PAPR performance of OFDM systems. Several related articles regarding the analysis of PAPR performance of OFDM and SC-FDMA were presented in [17-19]. Finally, an analytic formula for PAPR probability density function was derived for single carrier even M-QAM modulations in [20].

To sum up, nearly no studies have investigated the PAPR performance of single carrier transmission systems when adopting odd-bit QAM constellations. Therefore, in this paper, PAPR performance of DFT-SOFDM system when using both cross and rectangular odd-bit QAM constellations at different FFT sizes (Bandwidths) and QAM orders will be analyzed, simulated, tested and compared. Moreover, an expression of PAPR PDF for odd-bit cross QAM based DFT-S-OFDM systems will empirically be derived. Section 2 of this article will provide a theoretical analysis for different odd-bit QAM constellations distribution patterns and their impact on the PAPR performance of DFT-S-OFDM systems. The proposed model used to simulate and test the PAPR performance of odd-bit QAM based DFT-S-OFDM system will also be included in Section 2. Section 3 will introduce the simulation results represented by the PAPR PDF and CCDF curves for both rectangular and cross odd-bit QAM based DFT-S-OFDM systems at various FFT sizes, and constellation orders. It will also include the derivation of an empirical expression of PAPR PDF for odd-bit cross QAM based DFT-S-OFDM systems. Finally, the important conclusions will be presented in Section 4.

\section{RESEARCH METHODOLOGY AND THE PROPOSED MODEL}

Since the PAPR of DFT-S-OFDM system depends on the input symbols, Odd-bit QAM distribution patterns and required energy have a direct impact on the PAPR of the system. Hence, a theoretical analysis and derivations regarding odd-bit QAM different constellations and DFT-S-OFDM systems in terms of PAPR is crucial in the investigation of the PAPR performance of DFT-S-OFDM systems when adopting oddbit QAM.

\subsection{Odd-bit QAM}

Odd-bit QAM maps an odd number of bits (k) into $\mathrm{M}$ symbols where $\mathrm{k}=\log _{2} \mathrm{M}$. Many patterns were proposed as candidates for odd-bit QAM such as the rectangular, cross, circular or triangular constellations, yet, the most popular patterns used for Odd QAM are the rectangular and cross constellations. The odd-bit rectangular QAM constellation points are arranged in $2^{\mathrm{m}}$ columns and $2^{\mathrm{n}}$ rows where $\mathrm{m}=\mathrm{n}+1$. Figure 1 shows the odd-bit rectangular QAM of 8 and 32 size constellations.

As shown in Figure 1, rectangular odd-bit QAM uses gray labelling (gray penalty is 1) to map data bits into symbols in order to reduce BER. Moreover, it is easier to generate and demodulate the rectangular odd-bit QAM signal since it is represented by two pulse amplitude modulation (PAM) signals on phase quadrature carriers. However, it is obvious that the average power in real and imaginary parts is unbalanced leading to a high PAPR value that limits the performance of power amplifiers by driving them out of linearity.

Cross odd-bit QAM, as rectangular odd-bit QAM, maps an odd number of bits into symbols. Yet, the way of distributing the constellation points is different. As shown in Figure 2, the constellation points on 
the edges of the constellation diagram are moved up and down [21]. This arrangement gives the cross odd-bit QAM balanced average power between real and imaginary signals, thus, enhancing the PAPR performance of the system.

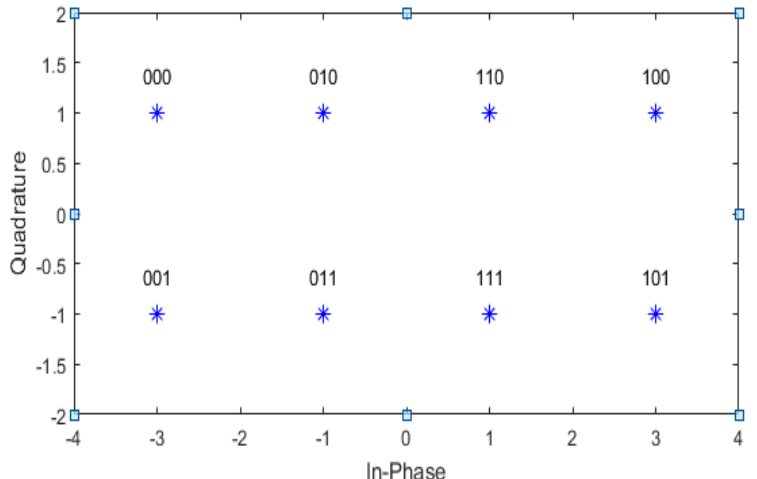

(a)

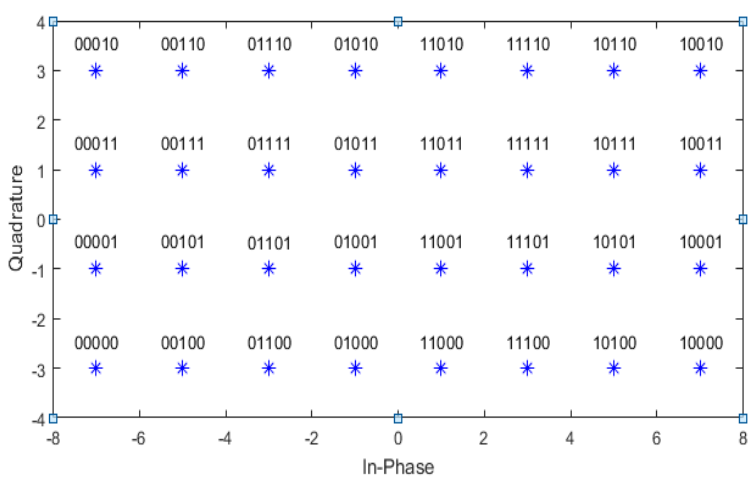

(b)

Figure 1. Constellation points distribution for (a) 8-Rectangular QAM, (b) 32-Rectangular QAM

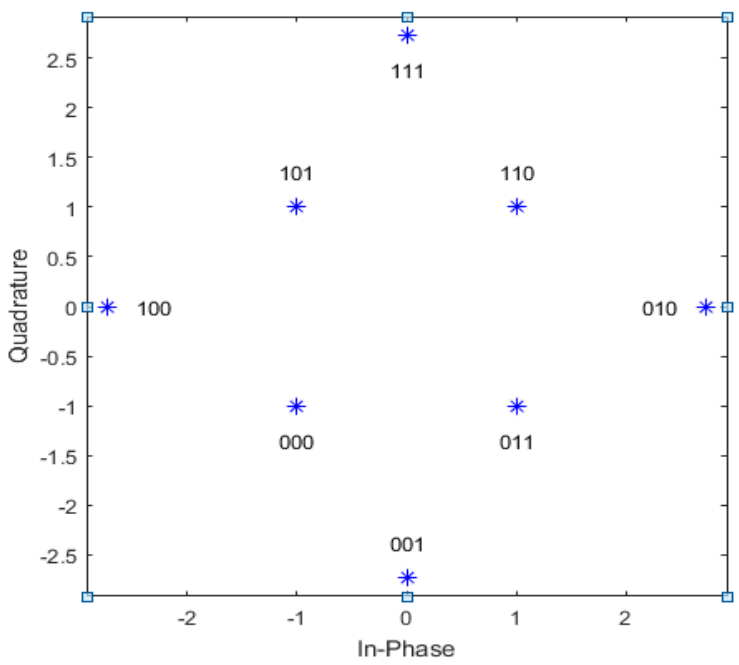

(a)

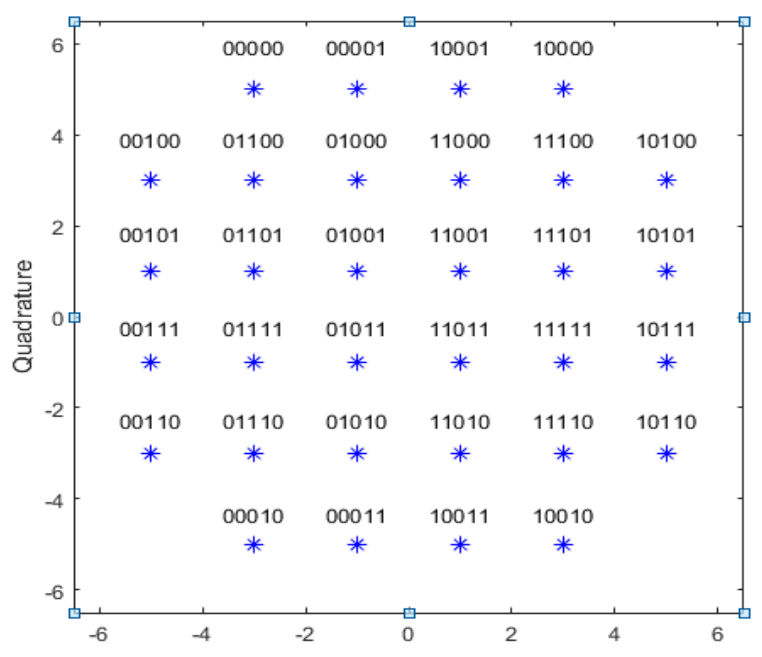

(b)

Figure 2. Constellation points distribution for (a) 8-Cross QAM, (b) 32-Cross QAM

Moreover, the cross odd-bit QAM surpasses rectangular odd-bit QAM in terms of required energy for a minimum Euclidean distance (dmin) of two as shown in (1).

$$
\begin{aligned}
& E_{a v}=\frac{1}{M} \sum_{n=1}^{M}(R n)^{2} \\
& E_{a v(8-R Q A M)}=\frac{1}{8}(4 \times 2+4 \times 10)=6 \\
& E_{a v(8-C Q A M)}=\frac{1}{8}\left(4 \times 2+4 \times(1+\sqrt{3})^{2}\right)=4.73 \\
& C Q A M \text { Gain }=10\left[\log _{10}(6)-\log _{10}(4.73)\right]=1.03 \mathrm{~dB}
\end{aligned}
$$

Applying the same approach for rectangular and cross 32-QAM constellations yields in CQAM gain of $1.14 \mathrm{~dB}$. As shown in Figure 2, cross odd-bit QAM adopts a pseudo gray labelling which unlike the rectangular odd-bit QAM, is an imperfect labelling that increases the BER. However, since the gray penalty for cross odd-bit QAM is near unity (10/8 for 8-CQAM and 7/6 for 32-CQAM) [21], the BER performance will slightly be affected by the adoption of cross odd-bit QAM constellations. 


\subsection{PAPR analysis of DFT spread OFDM systems}

Although OFDM has a high tolerance for multipath fading, inter-symbol interference, and good spectral efficiency, peak to average power ratio (PAPR) is one of the big issues that need to be solved in OFDM systems. The reason behind the high PAPR value in OFDM systems is due to the phase constructive addition of the signals in subcarriers leading to high peaks in the resulting OFDM signal [22].

For large Fast Fourier Transform (FFT) sizes (FFT size larger than 64 [4]), and assuming that the data symbols are statistically independent and identically distributed, the real and imaginary parts of the complex OFDM signal will converge to a Gaussian distribution according to Central Limit Theorem. The envelope of the OFDM signal will naturally follow the Rayleigh distribution. Assuming that the average power of the data symbols is normalized to one, the PDF will be formulated by [23, (2)].

$$
f_{r_{n}}(r)=\frac{r}{\sigma^{2}} e^{-\frac{r^{2}}{2 \sigma^{2}}}=2 r e^{-r^{2}}
$$
obtained

Where $2 \sigma^{2}=1$. In order to obtain the PAPR CCDF, the CDF of the maximum of Rn must be

$$
\begin{aligned}
& F C_{R \max }(r)=1-P(R \max <r) \\
& F C_{R \max }(r)=1-\left(P\left(R_{0}<r\right) \times P\left(R_{1}<r\right) \times \ldots P\left(R_{N-1}<r\right)\right) \\
& F C_{R \max }(r)=1-\left(1-e^{-r^{2}}\right)^{N}
\end{aligned}
$$

Where $P\left(R_{m}<r\right)=\int_{0}^{r} F_{R n}(u) d u . m=0.1 .2 \ldots . N-1$

From (5), it can be deduced that the PAPR performance of OFDM systems degrades when FFT size gets larger, thus, limiting the performance of the communication system. One of the solutions to enhance the PAPR performance is to use DFT-S-OFDM system instead of OFDM system. In DFT-S-OFDM, the data symbols are FFT spread prior to the conventional IFFT process used in OFDM. Virtually, assuming that FFT and IFFT share the same size, FFT and IFFT will cancel each other making the PAPR nearly as same as the PAPR of single-carrier systems [23]. Due to its enhanced PAPR performance, the DFT-S-OFDM was adopted in the Long-term evolution standards uplink. Figure 3 shows the block diagram of the DFT-SOFDM.

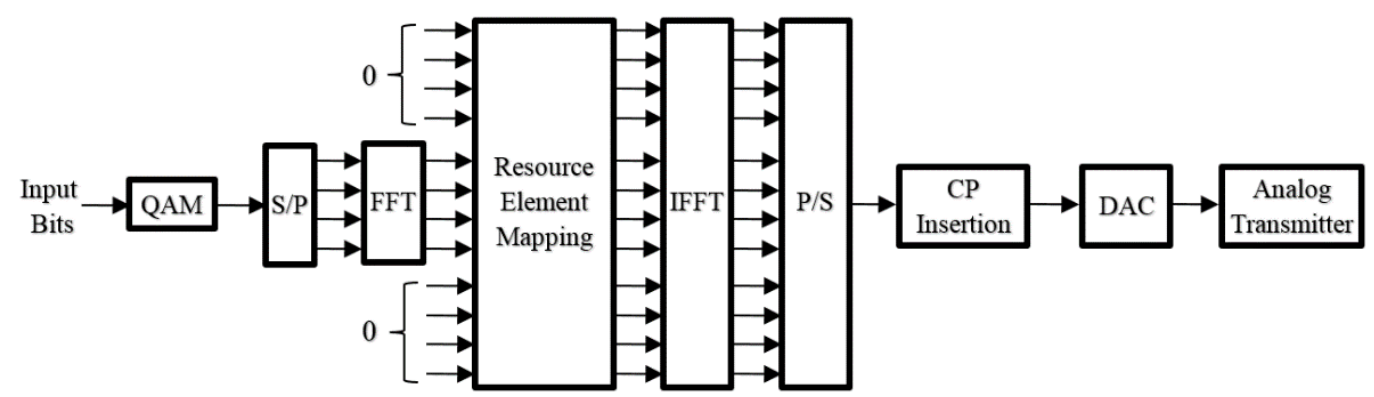

Figure 3. DFT-S-OFDM block diagram

Since DFT-S-OFDM system has approximately the same PAPR of a single carrier system, the choice of QAM constellation size or pattern has a significant impact on the PAPR performance of the communication system.

\subsection{The proposed model}

The proposed model focused on simulating the transmission process of many data packets through DFT-S-OFDM system when adopting both cross and rectangular odd-bit QAM schemes. Peak to average power ratio values for each transmitted DFT-S-OFDM symbol were measured and used in obtaining and visualizing PAPR PDF and CCDF curves. The detailed modelling procedure is stated in the following points. 
a) DFT spread OFDM system was simulated using MATLAB program. The simulation model was programmed to be configurable so that simulated communication system parameters such as FFT sizes, subcarriers mapping method, type of pulse shaping, pattern and size of the modulation scheme can be easily modified. Table 1 lists the parameters used for the simulation. LTE standard FFT sizes were adopted in the simulation.

Table 1. System simulation parameters

\begin{tabular}{cc}
\hline Parameters & Value \\
\hline Communication System & DFT Spread OFDM \\
Subcarrier Mapping & Localized \\
Pulse Shaping & Rectangular \\
FFT Sizes Tested & $128,256,512,1024,1536,2048[24]$ \\
QAM Patterns Tested & Cross, Rectangular \\
QAM Sizes Tested & 8-QAM, 32-QAM \\
\hline
\end{tabular}

b) The simulation involved sending a 50000 data packets of different legnths (depending on FFT size and the order of QAM modulation selected) through the simulated DFT-S-OFDM communication system. For each data packet transmission, we measured the power of of all complex symbols in the transmitted DFT-S-OFDM signal. Then, we obtained PAPR for each transmission (i) using [25, (6)].

$$
Y i=P A P R i(d B)=10 \log \left(\frac{\max \left(\left|S_{i k}\right|^{2}\right)}{\frac{1}{N} \sum_{k=1}^{N}\left(\left|S_{i k}\right|^{2}\right)}\right)
$$

Where $(\mathrm{S})$ is vector consisted of $(\mathrm{N})$ complex symbols that all together forms the transmitted DFTs OFDM signal. PAPR values for each transmission were stored in a separate vector denoted by $(\mathrm{Y})$.

c) A vector representing PAPR axis in PDF plot is created and denoted by (X) with $3 \mathrm{~dB}$ as minimum value, $13 \mathrm{~dB}$ as maximum value, and $0.05 \mathrm{~dB}$ step. From the PAPR values stored in vector (Y), we obtained PAPR probability density function and plotted it as a probability vector (PDF) versus (X) using the following relation.

$$
P D F(j)=f(X j)=P\left(X_{j} \leq P A P R \leq X_{j+1}\right)=\frac{\sum_{i=1}^{p k t} \operatorname{card}\left(Y i \leq X_{j+1}\right)-\sum_{i=1}^{p k t} \operatorname{card}\left(Y i \leq X_{j}\right)}{p k t}
$$

Where $\operatorname{card}\left(Y i \leq X_{j}\right)$ is the number of elements in vector (Y) where $\left(Y i \leq X_{j}\right)$, (pkt) is the number of data packets transmitted (50000 in our model).

d) PAPR cumulative complementary distribution functions (CCDFs) had also been obtained and plotted from vector $(\mathrm{Y})$ using the relation.

$$
C C D F(j)=F(X j)=P\left(P A P R \geq X_{j}\right)=\frac{\sum_{i=1}^{p k t} \operatorname{card}\left(Y i \geq X_{j}\right)}{p k t}
$$

e) We applied curve-fitting techniques on the PAPR PDFs curves obtained from the simulation to derive empirical formula that describe the statistical distribution of PAPR in odd-bit cross QAM based DFT-SOFDM systems. Figure 4 shows the flow chart of the proposed model

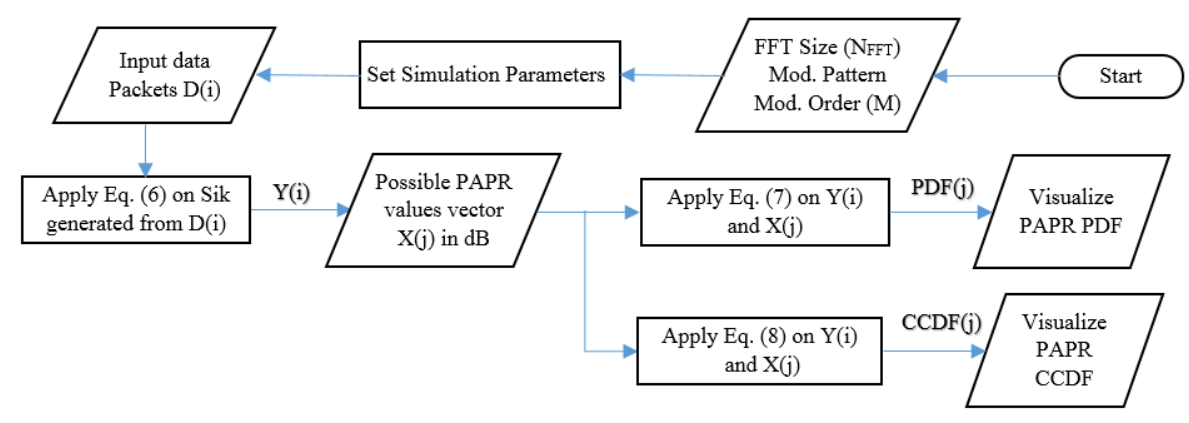

Figure 4. Flowchart of the proposed simulation model 


\section{SIMULATION RESULTS}

The system model described earlier had been simulated to test the PAPR performance of both cross and rectangular odd-bit QAM schemes for the FFT sizes, and QAM patterns specified in Table 1. Figure 5 shows the actual PAPR PDFs obtained from simulation (the dotted curves) for cross and rectangular odd-bit QAM constellations at the various FFT sizes and QAM orders mentioned in Table 1. PAPR PDFs also show that the mean $(\mu)$ is an increasing function of FFT size $(N)$ while the variance $\left(\sigma^{2}\right)$ is a decreasing function of $(\mathrm{N})$. Mean $(\mu)$ and variance $\left(\sigma^{2}\right)$ values for both cross and rectangular odd-bit QAM at all FFT sizes are measured and recorded in Table 2 and Table 3.

Table 2. Measured mean and variance values of 32-CQAM and 32-RQAM

\begin{tabular}{cccccccccccccc}
\hline \multicolumn{1}{c}{ 32-CQAM } & \multicolumn{10}{c}{32 -RQAM } \\
\hline $\mathrm{N}$ & 128 & 256 & 512 & 1024 & 1536 & 2048 & $\mathrm{~N}$ & 128 & 256 & 512 & 1024 & 1536 & 2048 \\
$\mu$ & 6.76 & 7.16 & 7.52 & 7.85 & 8.01 & 8.13 & $\mu$ & 7.92 & 8.40 & 8.82 & 9.20 & 9.39 & 9.52 \\
$\sigma^{2}$ & 0.58 & 0.44 & 0.34 & 0.27 & 0.23 & 0.21 & $\sigma^{2}$ & 0.73 & 0.55 & 0.44 & 0.34 & 0.29 & 0.26 \\
\hline
\end{tabular}

Table 3. Measured mean and variance values of 8-CQAM and 8-RQAM

\begin{tabular}{llllllllllllll}
\hline \multicolumn{1}{c}{ 8-CQAM } & \multicolumn{1}{c}{ 8-RQAM } \\
\hline $\mathrm{N}$ & 128 & 256 & 512 & 1024 & 1536 & 2048 & $\mathrm{~N}$ & 128 & 256 & 512 & 1024 & 1536 & 2048 \\
$\mu$ & 6.62 & 7.05 & 7.44 & 7.78 & 7.96 & 8.08 & $\mu$ & 7.59 & 8.08 & 8.48 & 8.82 & 9.00 & 9.13 \\
$\sigma^{2}$ & 0.66 & 0.51 & 0.40 & 0.32 & 0.27 & 0.25 & $\sigma^{2}$ & 0.66 & 0.50 & 0.38 & 0.29 & 0.25 & 0.23 \\
\hline
\end{tabular}

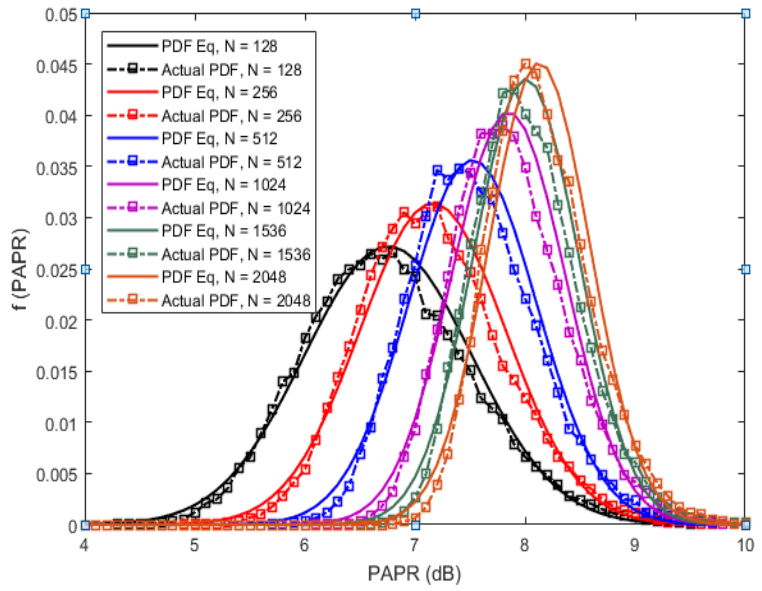

(a)

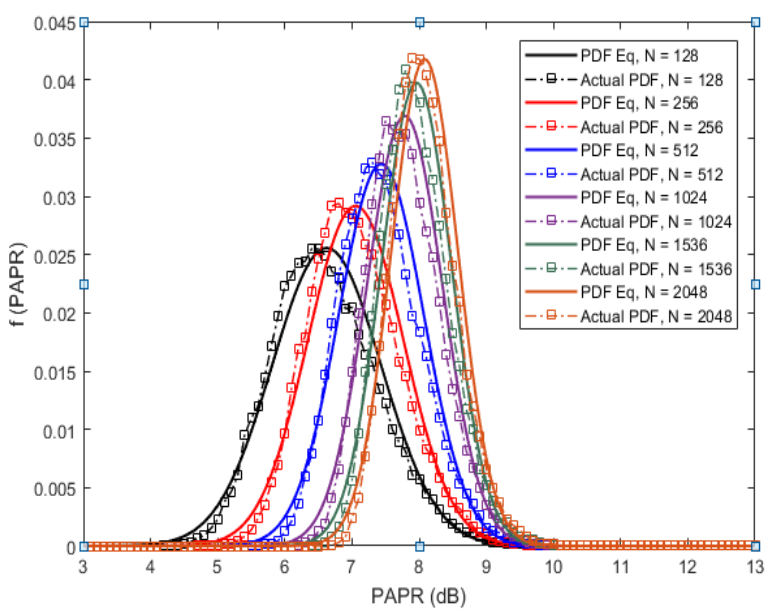

(c)

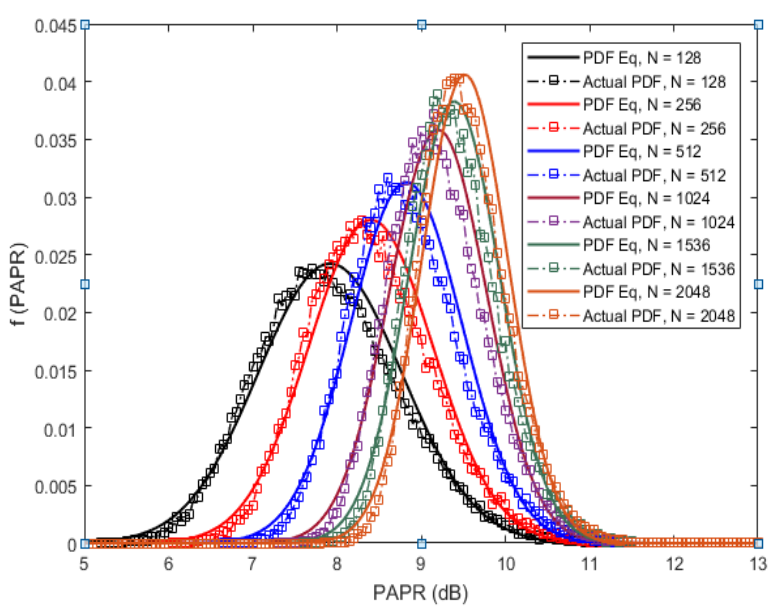

(b)

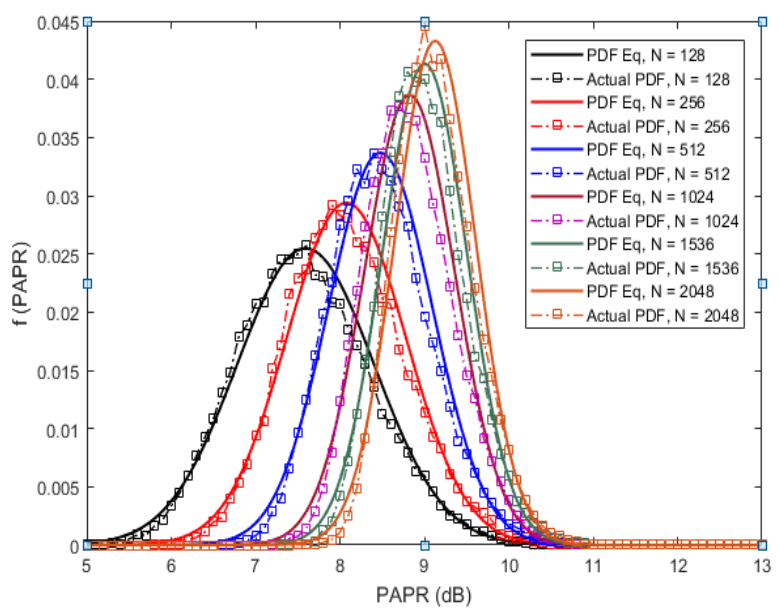

(d)

Figure 5. Actual PAPR PDFs based on simulation versus PAPR PDFs based on (12) for (a) 32-Cross QAM, (b) 32-Rectangular QAM (c) 8-Cross QAM, (d) 8-Rectangular QAM 
CCDF curves were also plotted for both cross and rectangular odd-bit QAM for 8, and 32 sizes at various bandwidths in Figure 6. The results confirm that cross odd-bit QAM outperforms rectangular odd-bit QAM by approximately the same margins obtained from the PDFs.

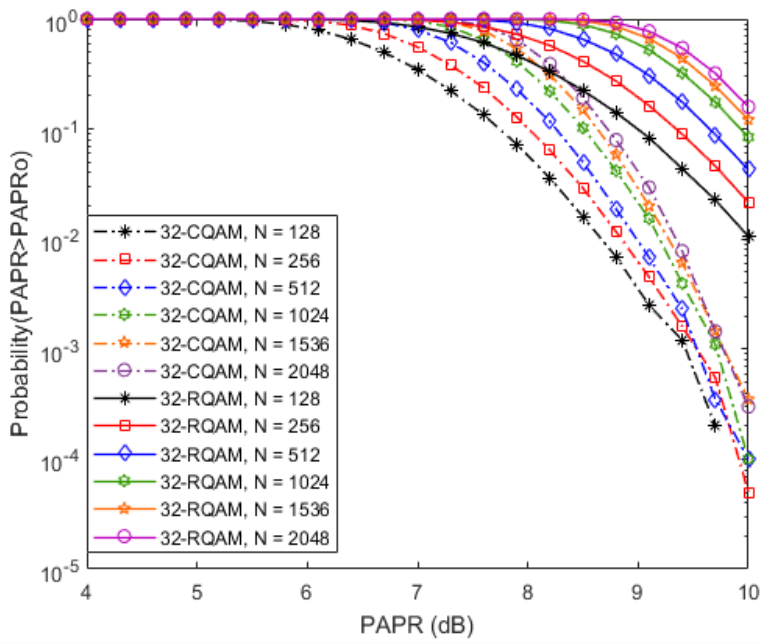

(a)

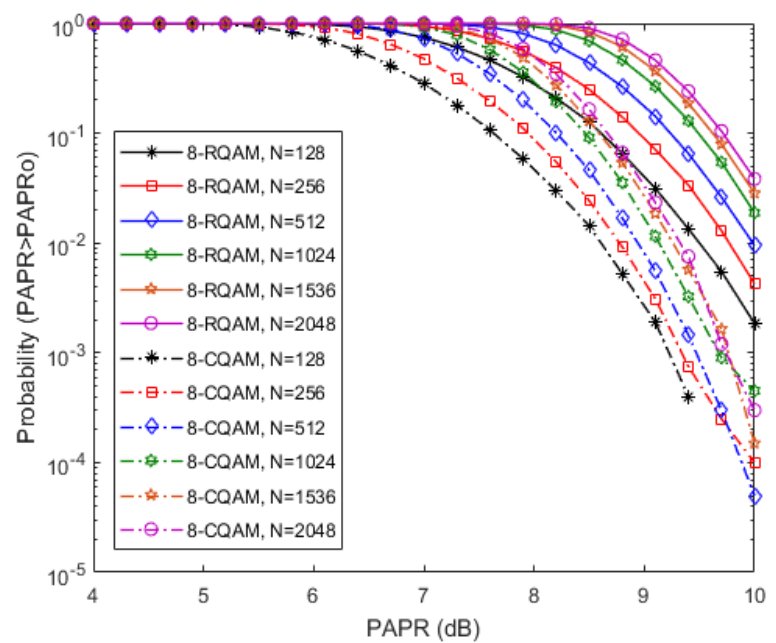

(b)

Figure 6. CCDFs of Cross QAM and Rectangular QAM for (a) 32-points size, (b) 8-points size

Gaussian curve-fitting technique (using MATLAB curve-fitting tool) had been applied on the PAPR PDFs vectors obtained from simulation in order to derive empirical formula that generally describes the PAPR statistical distribution of cross odd-bit QAM based DFT-S-OFDM system. The result of curve fitting shows that all PAPR PDFs when using cross odd-bit QAM schemes follow a scaled Gaussian distribution of (9).

$$
f(p)=\frac{0.052}{\sqrt{2 \pi} \sigma} e^{-\frac{(p-\mu)^{2}}{2 \sigma^{2}}}
$$

Where $(p)$ represents PAPR as random variable measured in $(\mathrm{dB}),(\mu)$ and $\left(\sigma^{2}\right)$ represents the mean and variance of the distribution.

As explained earlier, $(\mu)$ and $\left(\sigma^{2}\right)$ are increasing and decreasing functions of FFT size $(\mathrm{N})$ respectively. Power curve-fitting technique had been used to obtain a relation that describes $(\mu)$ and $\left(\sigma^{2}\right)$ as a function of FFT size $(\mathrm{N})$ using the measured values of mean and variance versus FFT size in Table 2 . The empirically obtained relations of $(\mu)$ and $\left(\sigma^{2}\right)$ as functions of FFT size $(\mathrm{N})$ are.

$$
\begin{aligned}
& \mu=4.86 N^{-0.07} \\
& \sigma^{2}=3.6 N^{-0.36}
\end{aligned}
$$

Thus, the final general form of PAPR PDF equation for cross odd-bit QAM based DFT-S-OFDM system as a function of FFT size would be.

$$
f(p)=\frac{0.052}{\sqrt{7.2 \pi N^{-0.36}}} e^{-\frac{\left(p-4.86 N^{-0.07}\right)^{2}}{7.2 N^{-0.36}}}
$$

The PAPR PDFs curves (the continuous curves) based on (12) in Figure 5 prove that the empirically derived PAPR PDF equation is highly accurate.

\section{CONCLUSIONS}

In this paper, the Peak to average power ratio (PAPR) performance of DFT-S-OFDM system is investigated, simulated, and tested while adopting cross and rectangular odd-bit QAM constellations. The 
results included PAPR PDFs and CCDFs for both rectangular and cross odd-bit QAM constellations at different FFT sizes (Bandwidths) and QAM orders. Curve fitting was applied on PAPR PDF curves obtained from simulation to empirically obtain a formula that accurately describes the PAPR statistical distribution when cross odd-bit QAM is used. Results showed the supremacy of cross odd-bit QAM over rectangular odd-bit QAM in terms of energy efficiency and PAPR performance.

\section{REFERENCES}

[1] Yiling Wu, Yuping Zhao, Hongbin Li, "Constellation Design for Odd-bit Quadrature Amplitude Modulation," 2010 IEEE Wireless Communication and Networking Conference Workshops, Sydney, NSW, pp. 1-4, 2010.

[2] Hideki Ochiai, Hideki Imai, "On the Distribution of the Peak-to-Average Power Ratio in OFDM Signals," IEEE Transactions on Communications, vol. 49, no. 2, pp. 282-289, 2001.

[3] Shuangqing Wei, et al, "A modern extreme value theory approach to calculating the distribution of the peak-toaverage power ratio in OFDM systems," 2002 IEEE International Conference on Communications. Conference Proceedings. ICC 2002 (Cat. No.02CH37333), New York, NY, USA, vol. 3, pp. 1686-1690, 2002.

[4] Tao Jiang, Mohsen Guizani, Hsiao-Hwa Chen. Weidong, Xiang, Yiyan Wu, "Derivation of PAPR Distribution for OFDM Wireless Systems Based on Extreme Value Theory," IEEE Transactions on Wireless Communications, vol. 7, no. 4, pp. 1298-1305, 2008.

[5] Tao Jiang, Yiyan Wu, "An Overview: Peak-to-Average Power Ratio Reduction Techniques for OFDM Signals," IEEE Transactions on Broadcasting, vol. 54, no. 2, pp. 257-268, 2008.

[6] P. Sivakumar, S. Sangeetha and M. Rajaram, "PAPR reduction in OFDM for SC-FDMA channels," 2011 International Conference on Signal Processing, Communication, Computing and Networking Technologies, Thuckafay, pp. 6-9, 2011. doi: 10.1109/ICSCCN.2011.6024504.

[7] Srinivas Ramavath, Rakhesh Singh Kshetrimayum, "Analytical calculations of CCDF for some common PAPR reduction techniques in OFDM systems," International Conference on Communications, Devices and Intelligent Systems (CODIS), Kolkata, India, pp. 393-396, 2012.

[8] A. P. More and S. B. Somani, "The reduction of PAPR in OFDM systems using clipping and SLM method," 2013 International Conference on Information Communication and Embedded Systems (ICICES), Chennai, pp. 593-597, 2013. doi: 10.1109/ICICES.2013.6508385.

[9] C. Rajasekhar, D. Srinivasa rao, V. Yaswanth Raghava and D. Hanith, "PAPR reduction performance in OFDM systems using channel coding techniques," 2014 International Conference on Electronics and Communication Systems (ICECS), Coimbatore, pp. 1-5, 2014. doi: 10.1109/ECS.2014.6892651.

[10] Guruprasad, Mohana and H. V. R. Aradhya, "PAPR reduction and performance analysis of modulation techniques in OFDM for WLAN applications," 2016 International Conference on Signal Processing, Communication, Power and Embedded System (SCOPES), Paralakhemundi, pp. 1567-1572, 2016. doi: 10.1109/SCOPES.2016.7955703.

[11] V. Montalvo, A. F. Reyes and M. C. Paredes-Paredes, "Comparison and analysis of PAPR reduction techniques in OFDMA and SC-FDMA systems," 2019 IEEE Fourth Ecuador Technical Chapters Meeting (ETCM), Guayaquil, Ecuador, pp. 1-6, 2019. doi: 10.1109/ETCM48019.2019.9014864.

[12] H. G. Myung, J. Lim and D. J. Goodman, "Peak-To-Average Power Ratio of Single Carrier FDMA Signals with Pulse Shaping," 2006 IEEE 17th International Symposium on Personal, Indoor and Mobile Radio Communications, Helsinki, pp. 1-5, 2006. doi: 10.1109/PIMRC.2006.254407.

[13] P. Li, Y. Zhu, Z. Wang and N. Wang, "Peak-to-average power ratio of SC-FDMA systems with localized subcarrier mapping," 2010 Global Mobile Congress, Shanghai, pp. 1-6, 2010. doi: 10.1109/GMC.2010.5634563.

[14] C. H. Yuen and B. Farhang-Boroujeny, "Analysis and optimization of PAPR in SC-FDMA systems," 2011 8th International Conference on Information, Communications \& Signal Processing, Singapore, pp. 1-5, 2011. doi: 10.1109/ICICS.2011.6174273.

[15] Ying-Che Hung, Shang-Ho (Lawrence) Tsai, "PAPR Analysis and Mitigation Algorithms for Beamforming MIMO OFDM Systems," IEEE Transactions on Wireless Communications, vol. 13, no. 5, pp. 2588-2600, 2014.

[16] Shukla, Jyoti, Alok Joshi, and Rajesh Tyagi, "PAPR analysis of OFDM system using AI based multiple signal representation methods," TELKOMNIKA (Telecommunication Computing Electronics and Control), vol. 17, no. 6, pp. 2983-2991, 2019.

[17] Vincent Savaux, Yves Lou"et, "PAPR Analysis as a Ratio of Two Random Variables: Application to Multicarrier Systems with Low Subcarriers Number," IEEE Transactions on Communications, vol. 66, no. 11, pp. 5732-5739, 2018.

[18] Hasan, Rawaa J., and Hikmat N. Abdullah, "Comparative study of selected subcarrier index modulation OFDM schemes" TELKOMNIKA (Telecommunication Computing Electronics and Control), vol. 17, no.1, pp. 15-22, 2019.

[19] S. P. Yadav and S. C. Bera, "PAPR analysis of single carrier FDMA system for uplink wireless transmission," 2015 10th International Conference on Information, Communications and Signal Processing (ICICS), Singapore, 2015, pp. 1-5, doi: 10.1109/ICICS.2015.7459941.

[20] Kouakou Kouassi, Guillaume Andrieux, Jean-Francois Diouris, "PAPR distribution for single carrier M-QAM Modulations," Wireless Personal Communications, vol. 104, no. 2, pp. 727-738, 2019.

[21] Pavan Kumar Vitthaladevuni, Mohamed-Slim Alouini, John C. Kieffer, "Exact BER computation for cross QAM constellations," IEEE Transactions on Wireless Communications, vol. 4, no. 6, pp. 3039-3050, 2005. 
[22] Ahmed M. Sana, Qasim Mohammed Hussein, Musa A. Hameed, Amer T. Saeed, "Performance analysis of circular 16-QAM constellation for single carrier-frequency division multiple access systems," Journal of Computational and Theoretical Nanoscience, vol. 16, no. 3, pp. 1019-1022, 2019.

[23] Yong Soo Cho, Jaekwon Kim, Won Young Yang, Chung G. Kang, MIMO OFDM wireless communications with MATLAB, Wiley Publishing, 2010.

[24] Zarrinkoub, H., Understanding LTE with MATLAB. New York: John Wiley \& Sons Inc, 2014.

[25] Proakis, J. G., and Salehi M., Fundamentals of Communication Systems Second Edition, Pearson Education, Inc., publishing as Prentice Hall, 1 Lake Street, Upper Saddle River, NJ 07458, 2014.

\section{BIOGRAPHIES OF AUTHORS}

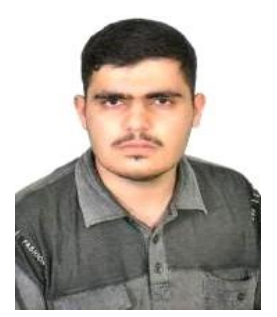

Ahmed M. Sana Received a B.Sc. degree in electrical engineering from Tikrit university, Iraq, in 2011 and M.Sc. degree in electronics and communications engineering from Baghdad university, Iraq, in 2014. In 2015, he worked as a power electronic engineer in the power department orient-telecom internet service company in Baghdad. Since 2017 and till now, He is working as a lecturer at Tikrit university, College of Petroleum Processes Engineering, Petroleum Systems Control department. His research interests include Power electronics, Digital Signal Processing, and 4G \& 5G communications systems.

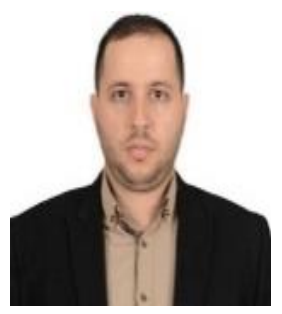

Amer T. Saeed Currently, he is an instructor at Tikrit University - College of Petroleum processes. He holds M.Sc. degree from the University of New Haven, USA in 2016 and received B.S. Degree in Electrical Engineering from the University of Tikrit-Iraq in 2009. He is Highly interested in 4G \& 5G communications systems, wireless networking and communications, Digital Signal Processing (DSP). He has published many papers in international and local conferences and journals.

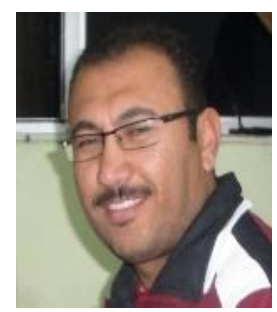

Yaseen Kh. Yaseen Received a B.Sc. degree in computer engineering from university of technology, Iraq, in 2000 and M.Sc. degree in computer engineering from university of technology, Iraq, in 2014. Since 2016 till now, He is working as a lecturer at Tikrit university, College of Petroleum Processes Engineering, Petroleum Systems Control department. His research interests include artificial intelligence, and computer communications. 